\title{
la photographie aérienne pour la reconnaissance de l'environnement
}

\author{
par \\ J. Aubert \\ Laboratoire Régional des Ponts et Chaussées, Melun \\ G. Gros \\ S.E.T.R.A., Paris
}

RESUME - Les auteurs veulent montrer comment en fonction de l'évolution de l'environnement au sens large et plus spécialement suivant les modifications: du cycle phytologique des végétaux, des états de surface des sols nus, du parcellaire agricole, il est possible de tirer d'une analyse multitemporelle des clichés verticaux des enseignements géotechniques propres à être transposés à l'étude d'un tracé routier ou d'une reconnaissance de gisement. De l'examen des couvertures photographiques établies dans le temps, il est possible de cerner les conditions d'apparition propres à un type d'indice donné ce qui permet alors de programmer une mission - verticale ou oblique - spécifique à un problème particulier dans un contexte géologique précis. Deux exemples sont proposés avec pour support un sol crayeux d'une part et des alluvions d'autre part. Une comparaison des résultats obtenus par une étude de photo-interprétation multitemporelle à partir de clichés verticaux et obliques et, par une prospection géophysique montrent les possibilités d'orientation d'une étude géotechnique.

"La photographie doit saisir la révélation inattendue et éterniser la fugitive" a écrit Aldoux HUXLEY. Or c'est bien souvent avec quelques secrets espoirs d'une découverte originale que le photo-interprète scrute un site nouveau ou encore peu connu. Si la révélation inattendue n'est pas alors éternisée, c'est peut être qu'elle était trop fugitive et que les conditions propices à sa saisie n'étaient pas réunies.

Aussi évoquerons-nous, dans un avant-propos introductif, les points essentiels de la démarche du photointerprète qui s'appuie sur l'analyse de l'environnement pris au sens large. Puis nous soulignerons $1^{\prime} \mathrm{e}-$ xistence d'archives photographiques nationales comme source d'informations d'une grande richesse. Dans une seconde partie, nous rappellerons l'intérêt de l'examen multitemporel en montrant, sur une zone précise, que la reconnaissance géotechnique ne saurait être pertinente par l'exploitation d'une seule mission fut-elle la plus récente. Nous indiquerons dans une troisième partie comment la somme des indices accumulés au cours de l'analyse de I'ensemble des missions couvrant un même site, mais réalisées à des époques différentes, conduit au choix des périodes favorables de prises de vues aériennes obliques à basse altitude sur des secteurs restreints pour 1 'examen détaillé du cadre physique et anthropique. Enfin nous établirons sur la base d'une comparaison des résultats obtenus, d'une part, grâce à la photo-interprétation et, d'autre part, au moyen de la prospection géophysique, que 1a photo-interprétation multitemporelle conjuguée à celle des prises de vues aeriennes obliques à basse altitude concoure efficacement à l'orientation de 1 a campagne de reconnaissance géotechnique de terrain.

I - AVANT-PROPOS INTRODUCTIF. \section{I.1 - Quelques aspects de la démarche du photo-}

En photogéologie, le photo-interprète tente de dégager des relations entre les éléments qui composent 1 'environnement et les phénomènes physiques, bio-physiques ou anthropiques. Pour cela, trois composantes sont à prendre en compte : I'environnement paysager, I'environnement cultural, I'environnement climatique.

Une fois établies, ces relations aboutissent à l'isolation d'indicateurs pertinents vis-à-vis de thèmes donnés. Ces indicateurs se répartissent en deux groupes : le groupe des indicateurs permanents et celui des indicateurs temporaires.

Le groupe des indicateurs permanents comprend ceux qui sont extraits de 1 'organisation du paysage dont les caractéristiques essentielles présentent une grande stabilité dans le temps comme celles contenues dans la morphologie du site et dans une moindre mesure son occupation.

Le groupe des indicateurs temporaires rassemble essentiellement ceux qui sont régis par 1'environnement climatique dont les variations marquent l'état hydrique du sol et l'état phytobiologique des cultures et végétaux, c'est-à-dire 1'environnement cultural.

Cette classification est étayée conme chacun sait par le fait que morphologie et occupation du sol, ainsi que leurs rapports mutuels, varient pour partie en fonction de la nature des terrains. Il s'ensuit qu'à partir de quelques points d'observation, il est aisé de déduire des caractéristiques géotechniques régionales. En voici trois exemples : 
zone boisées sur les buttes sableuses et siliceuses du tertiaire de 1'Est Parisien, forets sur les plateaux calcaires de l'Ouest Parisien, peupleraies dans les dépôts vaseux ou limoneux des vallées.

Toutes les informations à collecter ne vérifient pas de telles relations. Certaines, plus ténues, susceptibles d'être révélées par l'environnement cultural comme la présence de témoins archéologiques ou la fissuration d'un plateau calcaire dépendent alors de $1^{\prime}$ évolution climatique saisonnière et des conditions météorologiques locales qui fixent 1'humidité des sols et $1^{\prime}$ état phytobiologique du couvert végétal. Les indices qui en résultent apparaissent, selon les cas, dès la levée des semis ou bien seulement, par maturité différentielle, après l'épiaison. Ainsi sur sol alluvionnaire au droit d'un passage graveleux, les plants de céréales connaissent une densification plus faible et une maturation plus précoce que sur un encaissant plus limoneux ou plus argileux. Les plants de céréales seront alors exprimés par des teintes plus claires dans le premier cas. Les indices hydriques quant à eux sont fonction de la texture, de la granulométrie, de la porosité et de la nature chimique des sols. A la différence des indices phytobiologiques, ils sont beaucoup plus fugitifs car leur apparition est directement conditionnée par les précipitations et le vent. Ce dernier provoque du fait des hétérogénéités physico-chimiques locales des sols, un assèchement différentiel lequel en modifiant leur réflectance rend immédiatement compte de leur nature.

Ces divers indicateurs permanents et temporaires ne sont pas, par définition, visibles sur une seule et même mission. Leur présence pourra par contre en toute logique être attestée par une collection de photographies aériennes prises à des époques différentes. C'est cette chance qu'offre la photothèque nationale.

\section{I.2 - La photothèque nationale : une source d'une grande richesse d'informations.}

Le décret du 29 Mai 1946 instituant 1e centre de documentation photographique national (photothèque) stipule que celui-ci est chargé de rassembler les négatifs originaux ou à défaut les contre-types de toutes les photographies aériennes qui sont prises pour les besoins des services publics tant en métropole que dans les territoires et départements d'OUTREMER, exception étant faite pour les missions couvrant des surfaces inférieures à 1000 ha. Il est dans les attributions de ce centre d'assurer le classement et 1.a conservation des négatifs ou contre-types et de mettre cette documentation à la disposition de tous les départements ministériels en exécutant à la demande des services intéressés les tirages, agrandissements et assemblages nécessaires.

Depuis 1946 la photothèque a archivé 4 millions de clichés résultant d'émulsions panchromatiques, infrarouges noir et blanc et infra-rouges couleur. Certains de ces clichés ont été exécutés dans le cadre de la mission de vocation cartographique de 1'Institut Géographique National (I.G.N.). Les autres ont été réalisées par $1^{\prime} I . G . N$, ou d'autres prestataires pour des besoins divers pour le compte des services.

La couverture aérienne de la première catégorie est systématiquement stéréoscopique à une échelle homogène à $1 / 30000$ ou à $1 / 15000$ qui est fixée selon le type de cartes à élaborer. Elle est produite aux époques de 1 'année et aux moments de la journée les plus adaptés à la restitution photogrammétrique, c'est-à-dire en absence de végétation et d'ombre. En outre, il convient de noter que si la majeure partie de 1a FRANCE est aujourd'hui couverte de photographies prises au cours des 20 dernières années, dès 1981 la couverture aérienne du territoire national issue des travaux de vocation de $1^{\prime}$ I.G.N. devrait être telle que $I^{\prime}$ on pourra disposer en tout point de $1^{\prime}$ hexagone d'une couverture de moins de 5 ans soit au $1 / 30000$ soit au $1 / 15000$.

Par contre, la couverture de la deuxième catégorie rassemble aussi bien les missions à moyenne échelle commandées par le service de l'inventaire forestier national (I.F.N.) que celles plus ponctuelles stéréoscopiques ou non, aux échelles allant du 1/60 000 au 1/4000 faites à la demande des Directions Départementales et Services Techniques. Il s'ensuit une couverture dont les caractéristiques et le renouvellement sont très disparates puisque les émulsions, les dates et les échelles assurant le meilleur recensement forestier ne conviennent pas obligatoirement à la confection de plans à grande échelle ou à I'estimation des surfaces inondées.

Ainsi est mis à la disposition des services une importante documentation cartographique. Pourtant, la photo-interprétation des prises de vues aériennes verticales ne porte généralement, pour la reconnaissance des sites à aménager, que sur la plus récente couverture disponible. Que celle-ci provienne de la photothèque nationale, ou ait été spécialement effectuée pour satisfaire l'ensemble des besoins de l'étude d'un projet routier, elle ne s'avère pas toujours appropriée à une efficace exploitation pluridisciplinaire - archéologique - géotechnique - anthropique. C'est la raison pour laquelle il nous a paru utile de signaler, sur une zone précise, $1^{\prime}$ apport à la connaissance des sites de $1^{\prime}$ analyse systématique de clichés pris à des époques différentes.

\section{II - ANALYSE MULTITEMPORELLE.}

\section{II.1 - Présentation de la zone d'expérimentation.}

Il a été choisi dans le bassin parisien, au Nord du département de $1^{\prime} Y O N N E$, une zone d'expérimentation de $40 \mathrm{~km}^{2}$ (site $\mathrm{n}^{\circ} 1$, fig. 1).

Ce rectangle de $8 \mathrm{~km} \times 5 \mathrm{~km}$, se situe à $13 \mathrm{~km}$ au SudEst de la confluence SEINE-YONNE et s'étend entre les agglomérations de VILLENEUVE-1a-GUYARD, COURLON, VILLEMANOCHE et SAINT-AIGNAN. II est couvert par une dizaine de missions échelonnées entre les années 1949 et 1976. Cette zone est constituée de deux ensembles géotechniques : 1a vallée alluvionnaire de I'YONNE (alluvions silico-calcaires-gravelo-sableuses de 6 à $8 \mathrm{~m}$ de puissance), les collines crayeuses de la rive gauche avec, au Sud-Ouest, un recouvrement de plusieurs mètres de limons des plateaux et, au SudEst, la craie subaffleurante avec quelques placages épars de limon.

La photo-interprétation a porté sur des missions réalisées dans des conditions climatiques et météorologiques différentes telles que périodes arides (sécheresse de 1976) ou pluvieuses (inondations de 1955) et, par voie de conséquence avec couvert végétal très développé (printemps et été) ou très réduit (automne et hiver) (fig. 2). 

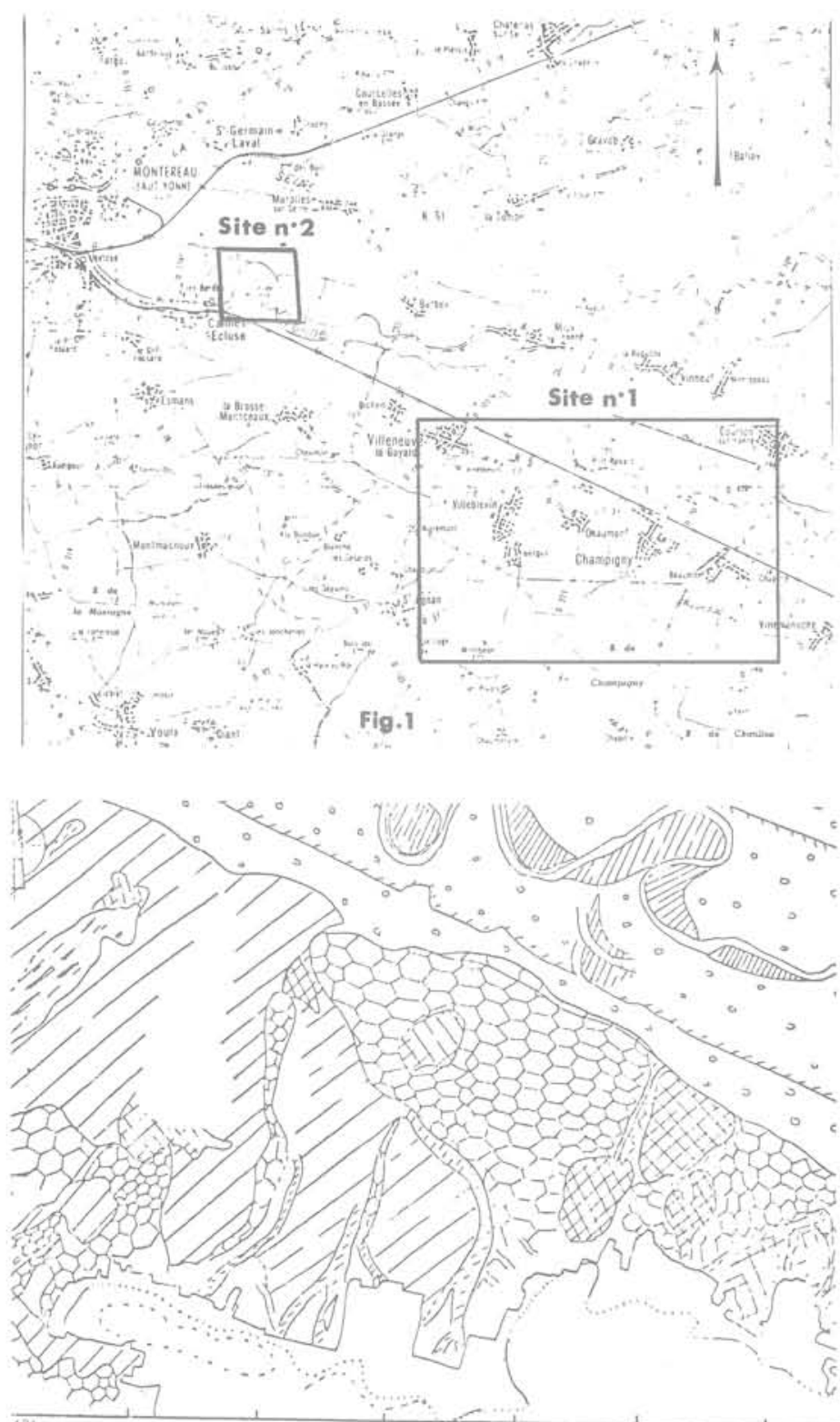

स्त्र Graie subattieurante $\square$ Ailuvioria acgilotimoneuses $\because$ Alluvions sablo-graveleuses $\square$ Limnordes plateaux

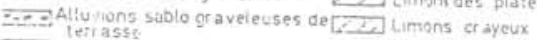

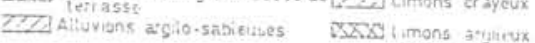

Fig. 2

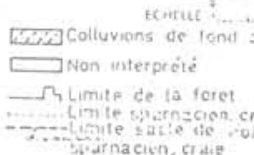

On relève, d'après le tableau I cing missions de printemps, trois missions d'été, une mission d'automne et une mission d'hiver.

\section{TABLEAU I}

\begin{tabular}{|l|l|l|}
\hline \multicolumn{1}{|c|}{ Epoque } & Echelle & \multicolumn{1}{c|}{ Emulsion } \\
\hline Aout 1949 & $1 / 25000$ & Panchromatique \\
Janvier 1955 & $1 / 25000$ & Panchromatique \\
Mai et Juin 1957 & $1 / 25000$ & Panchromatique \\
Mai et Juin 1961 & $1 / 25000$ et & Panchromatique \\
& $1 / 17000$ & \\
Octobre 1963 & $1 / 20000$ & Panchromatique \\
Avri1 1967 & $1 / 25000$ & Panchromatique \\
Printemps 1970 & $1 / 25000$ & Panchromatique \\
Mai 1969 & $1 / 25000$ & Panchromatique \\
Aout 1972 & $1 / 15000$ & Panchromatique et \\
Aout 1976 & $1 / 25000$ & Panchromatique \\
\hline
\end{tabular}

On s'est attaché à mettre en évidence l'intérét des couvertures les plus anciennes et à montrer 1'apport de chaque mission, d'une part, selon les indicateurs permanents fournis par la morphologie et 1 'occupation du sol et, d'autre part, en fonction des indicateurs temporaires associés aux diverses périodes de prise de vues.

\section{II.2 - Résultats acquis par 1'analyse multitem- porelle}

II.2.1 - Exploitation des données de 1'environnement paysager à partir des couvertures les plus anciennes.

L'exploitation des couvertures les plus anciennes de 25 ou 30 ans d'age fait apparaŝtre une évolution du paysage venant masquer les relations existantes avec la nature du sous-sol. Celle-ci concerne la modification des strates historiques du paysage par désorganisation des structures agraires anciennes dont 1'élaboration dans le cas présent, remonte au moyen-age. Ceci est da aux remembrements agricoles de 1a deuxième moitié du deuxième millénaire. Force est de constater que le découpage du territoire en parcelles agricoles n'obéit plus aujourd'hui aux mêmes règles qu'autrefois. Cette évolution imposée par les impératifs d'une exploitation rationnelle des terres efface du même coup le lien qui existait entre substrat et parcelles. En effet, celles-ci étaient bien souvent disposées en fonction de la nature des terrains et constituaient alors de précieux indices.

En voici deux exemples : le premier se rapporteà la Division de 1 'espace de 1 a zone des collines crayeuses et celle des dépôts limoneux, dont la limite transparaissait encore dans le paysage en 1949. En effet, dans la zone de craie subaffleurante les pièces culturales destinées à la polyculture montraient des dimensions restreintes et des superficies moyennes de 1 'ordre de 10 à 15 ares. Par contre, dans la zone des dépats limoneux, les surfaces atteignaient 2 à 4 hectares. Aujourd'hui, la disharmonie tend à disparaitre par regroupement, mais figure encore dans la toponymie (cf, les grandes pièces).

Le second exemple est relevé dans la plaine alluviale où $1 \mathrm{a}$ modification de géométrie des parcelles oblitère quasi complétement la distribution spatiale des dépôts alluvionnaires (figs. 3 et 4 ).

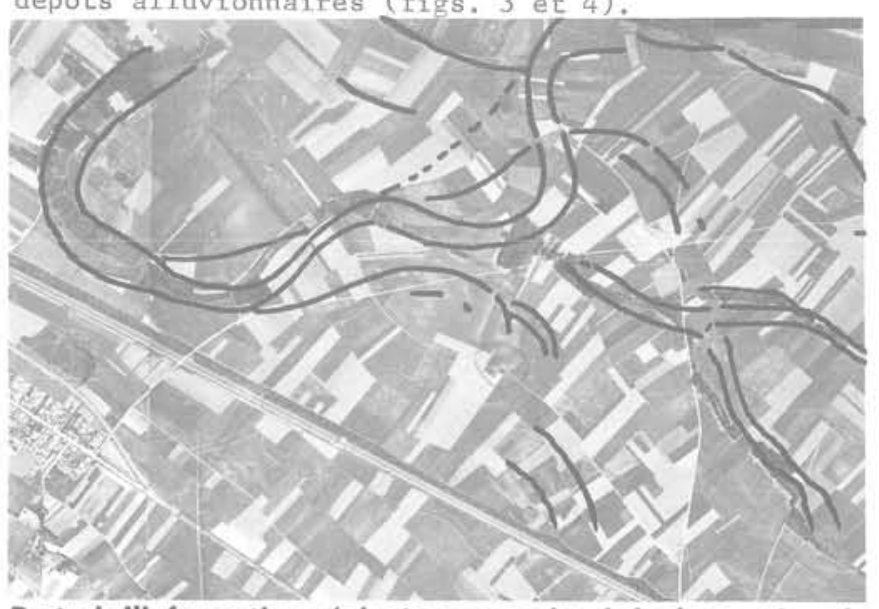

Perte de I'Information géologique par suite de la destruction du paysage

$=$ Indices originals en liaison avec le parcellaire ancien

(Couverture 1949) et conservés (Couverture 1969)

$z=z=$ Indices disparus apris remembrement agricole 


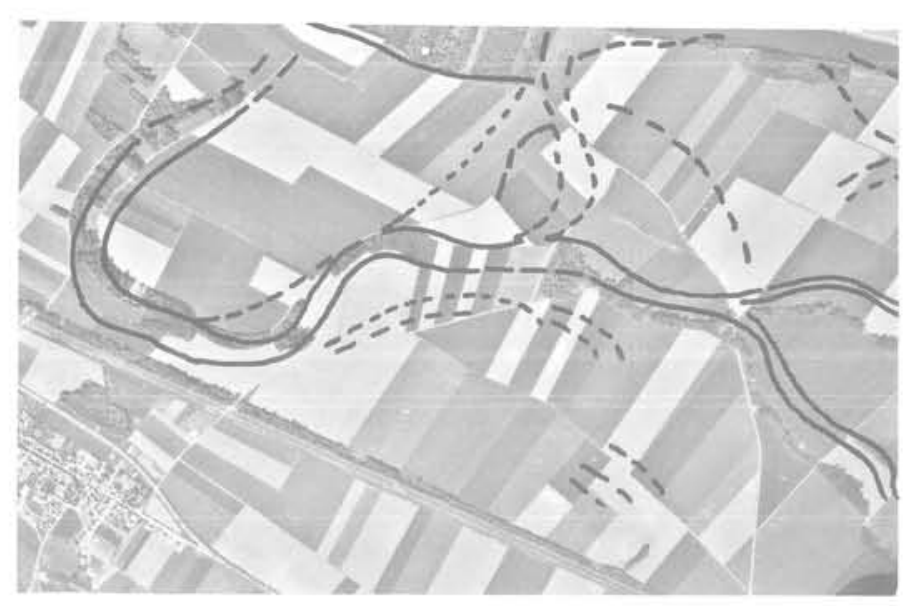

Perte de l'Information góologique par suite de la destruction du paysage

\section{$=$ Indices originals en liaison avec le parcellaire ancien \\ (Couverture 1949) et conservés (Couverture 1969) \\ :z:= Indices disparus apros remembrement agricole \\ Fig. 4}

La disposition courbe ou rayonnante des champs reflétait assez fidèlement les différenciations lithologiques des nappes de dépôts alluvionnaires en $s^{\prime}$ appuyant sur d'infimes variations de relief et de nature des sols. L'organisation alors retenue associée à une culture en billons favorisait l'écoulement des eaux superficielles et tendait vers un assainissement naturel des terres. Aujourd'hui, une nouvelle organisation est imposée qui implique dans les zones les plus argileuses la pose de drains artificiels.

Ces deux exemples illustrent clairement une dégradation de l'information géologique qui ne pourra que s'accentuer au fil des ans à mesure de la réalisation des programmes de remembrements ruraux et de tous les travaux connexes. C'est encore plus vrai dans des régions à fort développement urbain. Les témoins du passé sont éliminés par les exigences du monde moderne. L'historien aura perdu les raisons de $L^{\prime}$ 'organisation agraire d'antan et le géotechnicien l'expression de 1 'hétérogénéité des sols.

Cette perte de 1'information se trouve palliée en partie par les archives de la photothèque nationale et démontre tout $1^{\prime}$ intérêt de $1^{\prime}$ analyse multitemporelle des missions.

\section{II.2.2 - La complémentarité des missions.}

Plus qu'un long texte, la comparaison de la carte de synthèse des indices géologiques relevées sur l'ensemble des missions à deux des cartes dressées respectivement pour la mission de 1961 et celle de 1976 met bien en évidence 1 'apport complémentaire des diverses missions (figs. 5, 6 et 7 ). Du même coup, cela souligne les limites de la photo-interprétation portant sur une seule mission puisque l'exemple présenté porte sur deux missions qui ne sont pas les moins riches. D'une façon plus générale les tableaux II et III donnent une idée d'ensemble de la pertinence de chacune des missions quantifiant la qualité des indices relevés vis-à-vis des différentes catégories de terrain et d'environnement cultural.
CARTE SYMTHETICUL DES INDICES GEOLOGIQIIFS

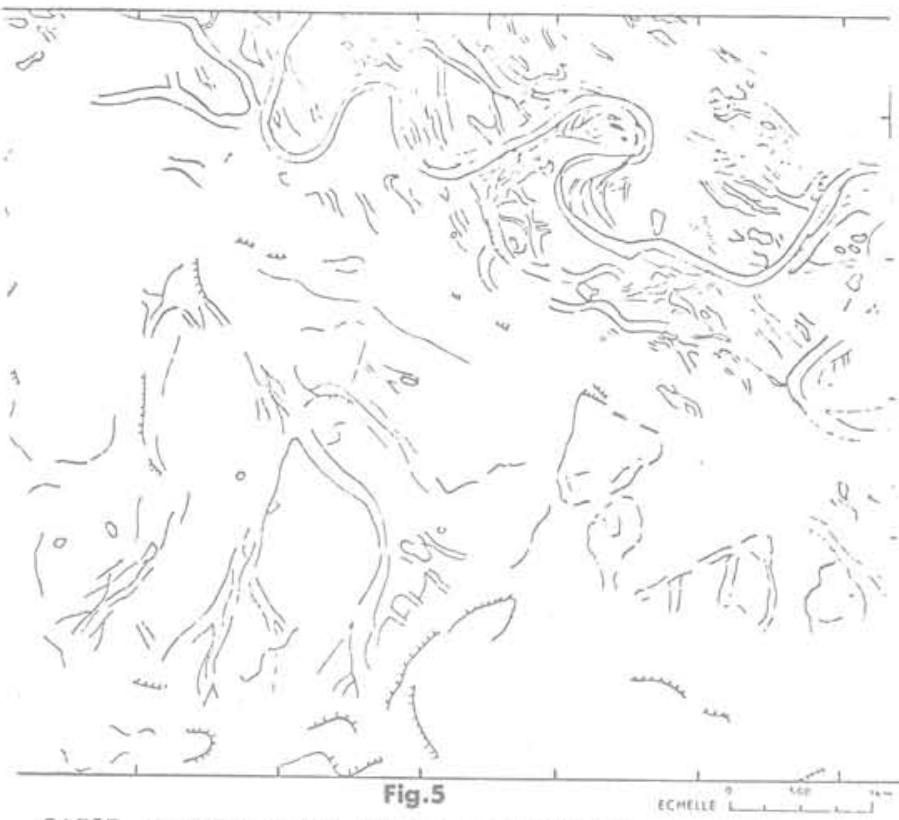

CARTE ANALYTIQUE DES INDICES GEOLOGIQUES
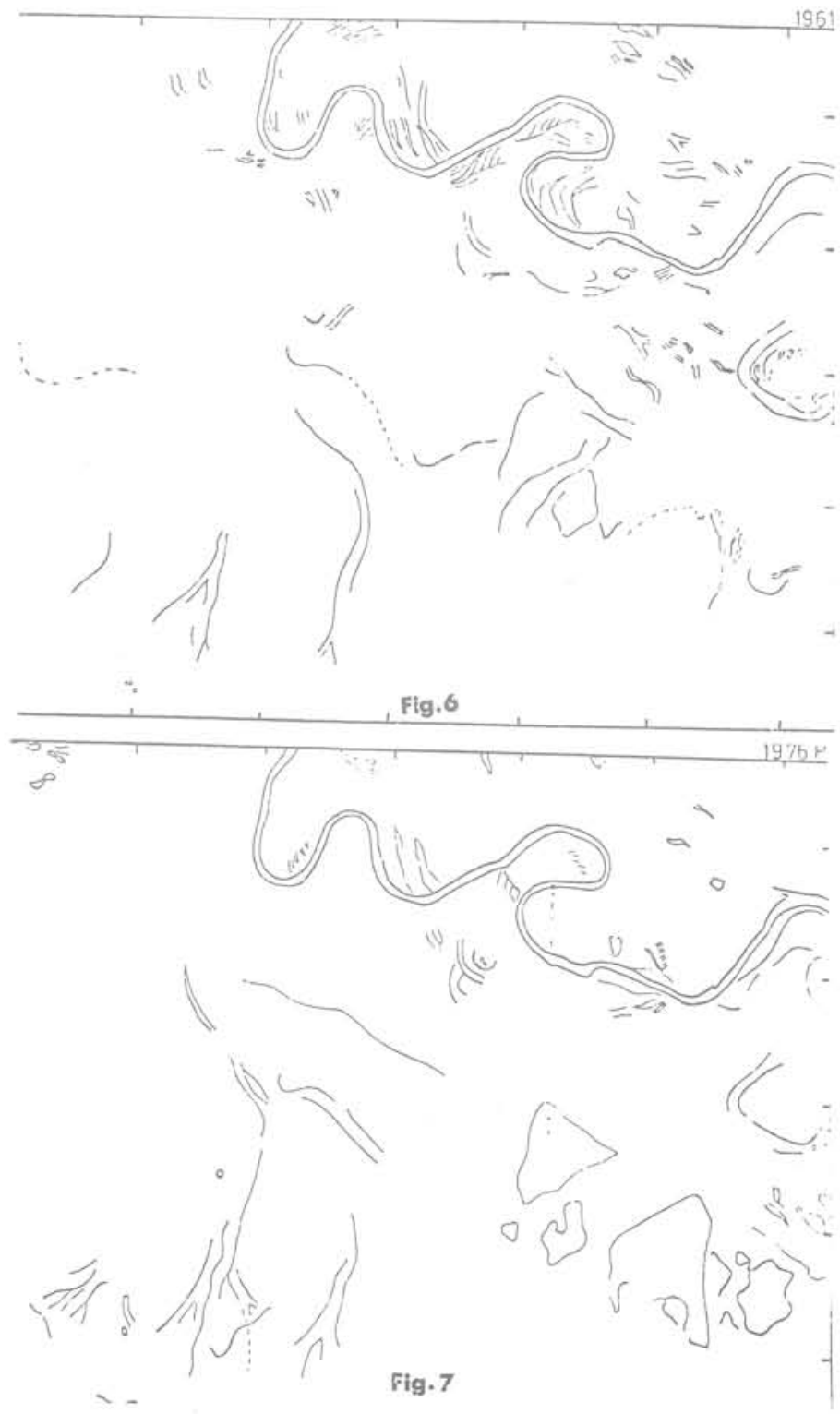
TABLEAU II

\begin{tabular}{|c|c|c|c|c|c|c|c|}
\hline & & crovie & de tis. at & cellaviona & at ivitione & purveflains & surnieree \\
\hline tsik - If Jwsier & 01.9 & couner & it carcititie & & 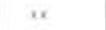 & & \\
\hline $\begin{array}{c}1250-2 e \text { nat } \\
\text { at juin }\end{array}$ & 01.2 & $x$ & $x$ & $x \times$ & $x \times x$ & $x \times x$ & $x$ \\
\hline tyeg - 18 dantier & 01.20 & Eouver & on portinili & & $x \times x$ & & \\
\hline $\begin{array}{l}\text { 1861-30 asi } \\
\text { a: juin }\end{array}$ & pl. 3 & $x$ & $\mathrm{xx}$ & $x$ & $x \times x$ & & \\
\hline $7808-10$ artobre & 01.6 & $x \times x$ & $x \times x$ & $x x$ & & $x \mathrm{xx}$ & $x \times x$ \\
\hline 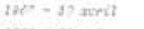 & $p 1.5$ & $x^{x}$ & $x x$ & ceuvertur & Dartiolio & $m x$ & \\
\hline 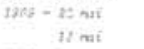 & 01.6 & $x$ & $x$ & $=$ & & , & $x$ \\
\hline $1520=7$ & & $x \times x$ & $\mathrm{xxx}$ & cenowartur. & Dartseise & $\mathrm{xx} \times \mathrm{x}$ & \\
\hline th/z -5 ancit & pl.z $=1$ fis & $x$ & $x$ & $x$ & & $x$ & x \\
\hline $12 x-8$ dost & pt $\theta$ et & $x x$ & $x \times x$ & $x \times x$ & $x$ & $x=$ & $x$ \\
\hline
\end{tabular}

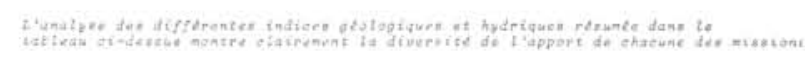

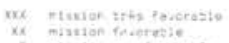

TABLEAU III

INDICES EN FONCTION DE L'OCCUPATION AU SOL

\begin{tabular}{|c|c|c|c|c|c|c|c|c|c|}
\hline \multicolumn{2}{|c|}{ 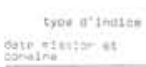 } & \multicolumn{2}{|c|}{ 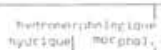 } & \multicolumn{2}{|c|}{ 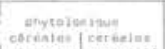 } & chiomente & 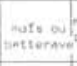 & $\begin{array}{l}\text { picromor: } \\
\text { phivilostaun }\end{array}$ & $\begin{array}{l}\text { potosin } \\
\text { siloan }\end{array}$ \\
\hline & $\begin{array}{l}\text { alivionot } \\
\text { envie }\end{array}$ & ${ }_{k x}^{x}$ & $y X$ & & & & & f & \\
\hline $195^{\circ}$ & 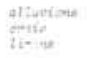 & & & $\underset{x}{x x}$ & & & & & \\
\hline $\mid P S P$ & 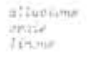 & $\underset{x y}{x x}$ & IXI & & & & & & \\
\hline 1961 & 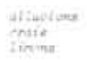 & & & & $\begin{array}{l}x x \\
x \\
x\end{array}$ & & & & 7 \\
\hline 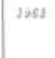 & 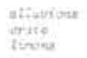 & $x_{x X}^{x x}$ & & & & $x$ & & $X X X$ & $\begin{array}{l}x \\
x x \\
x x x\end{array}$ \\
\hline 1986 & $\min _{\operatorname{tin} 0}$ & $x x$ & & IS & & & & & \\
\hline 1569 & $\begin{array}{l}\text { atturient } \\
\text { Tuice } \\
\text { tumat }\end{array}$ & & & $\frac{x}{x}$ & & & & & \\
\hline $3 x \geq 2$ & $\begin{array}{l}\text { athuviant } \\
\text { creve } \\
\text { tinonit }\end{array}$ & & & & $x$ & & $\frac{x}{x}$ & & \\
\hline 1870 & $\begin{array}{l}\text { Altwerions } \\
\text { ericie } \\
\text { tinons }\end{array}$ & $\begin{array}{l}x x x \\
x x x\end{array}$ & & & & $\begin{array}{l}x x \\
x x\end{array}$ & $\begin{array}{l}x x x \\
X X X \\
X X X\end{array}$ & & \\
\hline
\end{tabular}

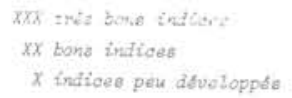

De 1 'analyse de tous ces indices, il ressort que pour une période de prise de vua déterminée, il n'est pas possible de trouver apparemment un moment favorable unique pour 1 'ensemble des observations géologiques et géotechniques.

Les hétérogénéités alluvionnaires sont le mieux perçues en mai-juin, c'est-à-dire à une période où les céréales parviennent à une maturité différentielle.

La recherche de lits mineurs et de champs actifs demande à bénéficier de conditions de très hautes eaux (inondation décennale ou centenaire).

L'analyse du recouvrement limoneux montre que le printemps voire $1^{\prime}$ automne avec le maximum de sols nus constituent une bonne période, mais que de bons résultats peuvent être acquis en été dans des conditions d'extrême aridité.

L'infra-rouge apporte des précisions très utiles sur 1 'humidité de surface, le rabattement de nappe, les zones de rétention, la cartographie des plans d'eau, dans certains cas toutefois 1'émulsion panchromatique peut être supérieure pour 1'étude par le biais d'indices phytologiques.
Toutes les informations examinées n'apportent pas, pour le site étudié, la même richesse d'informations. En effet, sur la dizaine de couvertures consultées, deux sont dépourvues presque totalement de renseignements (1969, 1972), trois seulement auraient suffi à 1a cartographie des alluvions (1955, 1961, 1976), deux enfin apparaissent efficaces pour 1 'étude des affleurements crayeux et du recouvrement limoneux (1963-1976). Autrement dit, seulement quatre missions convenablement choisies (1955, 1961, 1963, 1976) fournissent 1 'essentiel de 1 'information pour 1 'établissement d'une carte de synthèse géotechnique.

S'il n'existe donc pas une période de prise de vues favorable à la mise en évidence de l'ensemble des indices, il est clair que selon l'objectif poursuivi par le photo-interprète, le choix des missions doit être guidé par les dates de prises de vues. En 1'occurrence, mai-juin pour les problèmes alluvionnaires, automne et printemps pour les régions crayeuses. Par ailleurs, des conditions exceptionnelles, sécheresse par exemple ou au contraire inondations d'hiver, valorisent les clichés, mais dans ce dernier cas les facteurs extérieurs, pluie, brouillard, nuages peuvent entraver les vols à haute altitude. En outre, 1 'exploitation systématique de la documentation existante permet d'appréhender les indices susceptibles d'être décelés et facilite ainsi le choix des périodes de prises de vues adaptées à leur saisie exhaustive au moyen de la photographie aérienne oblique à basse altitude.

\section{III - SUIVI DES INDICES DANS LE TEMPS PAR LA PHOTO- GRAPHIE AERIENNE OBLIQUE.}

Sur la base des résultats précédents 11 a été entrepris le suivi des indices géologiques et archéologiques, d'une part, en terrain crayeux sur sols nus et, d'autre part, en dépôts alluvionnaires au travers du couvert végétal. Une série de prises de vues à basse altitude réalisée entre mars et août 1980 devait préciser la durée de visibilité de ces indices (fig. 8).

\section{1 - Etude des sols crayeux}

Cette étude indique que dès le mois de mars 1980 , après une période relativement chaude, pluvieuse et venteuse pour la saison, les indices géologiques et archéologiques se présentent comme suit (fig. 8) ;

- indices géologiques : ils offrent une très bonne visibilité globale avec une différenciation aisée des placages limoneux et des fonds de vallées colluvionnées. Ils demeurent visibles quatre semaines durant, soit sur sols nus, soit au travers de la levée des céréales. Toutefois, les anciennes manières très apparentes lors des céréales naissantes, le 3 mars, avaient totalement disparues, le 7 mars pour ne réapparâ̂tre, que très partiellement ( $20 \%$ des indices), lors de la maturité différentielle de céréale en juin.

- indices archéologiques : pendant le mois de mars, ils ne transparaissent pas ou peu dans les labours alors qu'ils sont identifiables sur les céréales. I1 s'agit essentiellement de fosses néolithiques et protohistoriques dont la persistance de la visibilité sur les céréales naissantes s'explique par la température moyenne relevée en janvier et février. Cette température est de $1^{\prime}$ ordre de 3 à $4^{\circ} \mathrm{C}$ supérieure à 

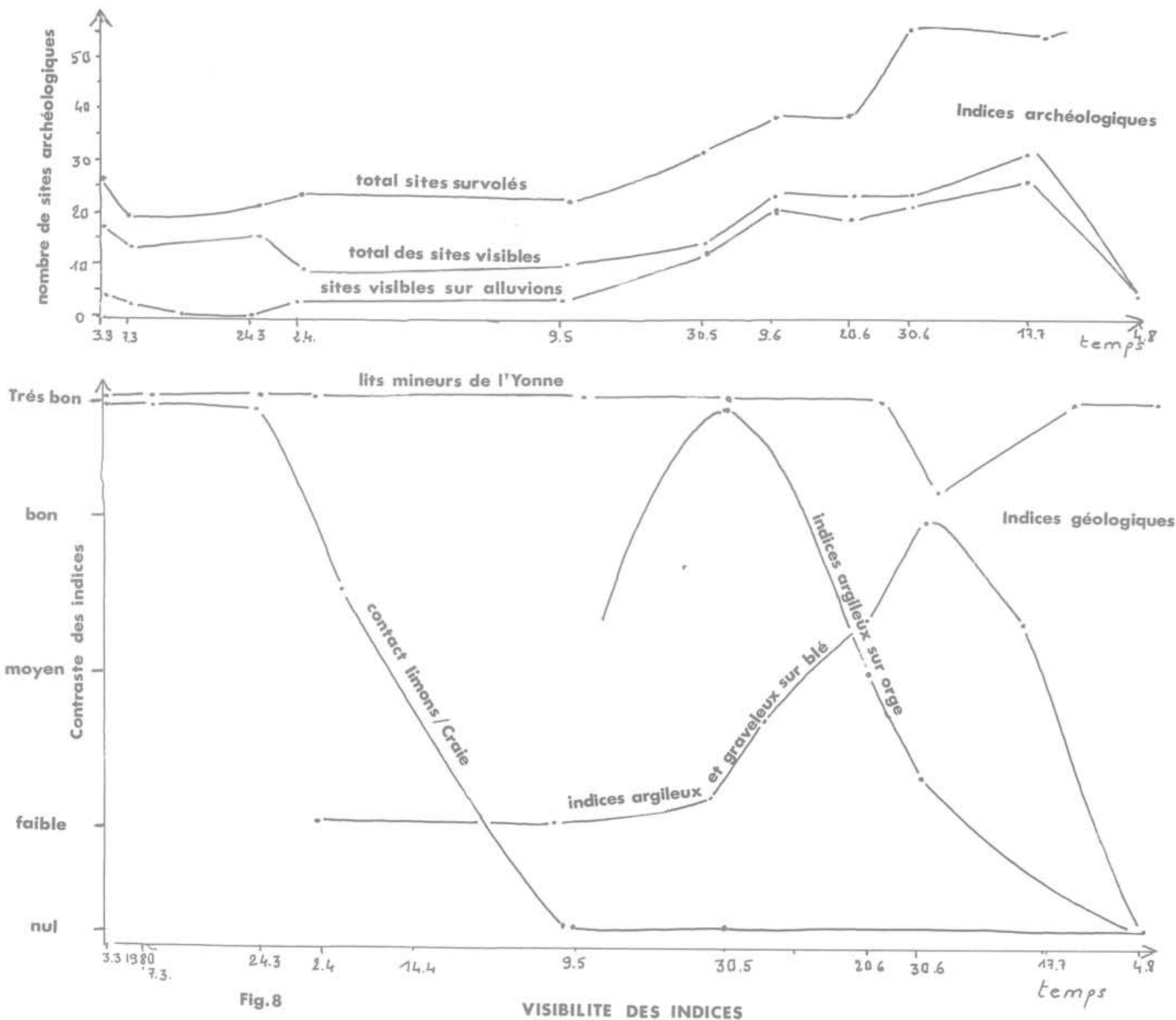

celle de 1'année précédente pendant laquelle il n'y avait eu aucun indice visible sur cette même zone. Autrement dit leur détection est liée à l'état phytobiologique des cultures. Qu'il s'agisse d'indices géologiques ou archéologiques la seule période favorable est celle de la fin de l'hiver pour les zones crayeuses.

\section{2 - Etude des dépôts alluvionnaires}

L'étude des dépôts alluvionnaires a essentiellement porté sur les matériaux graveleux et argileux (fig.8)

- indices géologiques : plusieurs cas sont à considérer :

- noues et lits mineurs : on a observé une visibité continue de mars à aoot 1980 qui s'explique dans les zones basses par la submersion préalable survenue fin février et début mars. Il en est advenu par excès d'eau un flétrissement et dépérissement des céréales d'hiver et une remise en culture à partir du mois de juin. C'est ce qui a permis de différencier la trace de
- matériaux graveleux : ils restent invisibles de mars à avril et offrent un bon contraste le 20 mai et au début aoat exception faite pour les parcelles moissonnées. En effet, les orges et les blés arrivés à maturité plus précocement entrainent une inversion des teintes sur les clichés panchromatiques noir et blanc. Ceci souligne l'influence de l'évolution phénologique du couvert cultural dont il importe de tenir compte pour la recherche d'indices en 1 'occurrence graveleux.

- matériaux argileux : il semblerait que la période de visibilité soit réduite à 8 jours par an avec au cours des mois de juin et juillet de brefs mais étonnants contrastes discontinus dans le temps.

- indices archéologiques : ceux-ci se comportent différemment avec les orges d'hiver et les blés. Avec les orges la durée d'observation des indices varie de 8 à 15 jours alors qu'avec les blés, l'observation reste possible pendant 2 mois (juin et juillet). 
On a pu constater au droit des fossés une teinte des végétaux plus accentuée et à la verticale d'autres vestiges une croissance différentielle précédée également par une différence de couleur au début. I1 convient de rapprocher ces remarques du déficit en eau de l'année 1980 qui s'élève, dans les parties hautes du site, à $80 \%$ en avril et $50 \%$ en mai par rapport à une année moyenne. De ce fait l'année 1980, dans un contexte alluvionnaire, présente pour la photo-interprétation les mêmes avantages que 1'année 1976 avec toutefois un déficit en eau plus précoce.

D'une façon globale le suivi régulier des indices a confirmé le schéma mis en évidence par la photo-interprétation multitemporelle. Il a précisé la durée de vie des indices géologiques et archéologiques ce qui devrait permettre, en association avec un suivi régulier des conditions météorologiques locales (par le biais du bilan hydrique intégré), de déclencher des missions aériennes ponctuelles avec le maximum de chances de réussite.

IV - CORRELATION DES INFORMATIONS OBTENUES PAR PHOTOINTERPRETATION AVEC LES CARACTERISTIQUES GEOTECHNIQUES DES MATERIAUX - RECONNAISSANCE DES GISEMENTS ALLUVIONNAIRES.

La photo-interprétation relève différents types d'indices soit du ler ordre lorsqu'il s'agit d'observations faites sur sols nus, soit du 2 ème ordre Iorsque 1 'analyse porte sur les modifications du couvert végétal. De telles observations seront d'autant plus utiles qu'elles pourront être reliées aux caractéristiques géotechniques des terrains. Aussi avons-nous procédé à la comparaison, d'une part, sur le site $n^{\circ} 1$ (présenté antérieurement) des indices mis en évidence et de la granulométrie des sols et, d'autre part, sur un autre site de la vallée de l'yONNE aux résultats obtenus par prospection électrique.

\section{IV.1 - Etude granulométrique comparative - site}

Du point de vue géotechnique la différence entre les sols graveleux et limoneux porte à $1 \mathrm{a}$ fois sur la variation d'épaisseur de la couche superficielle, sur une différence de nature et de granulométrie des formations sous-jacentes (sols fins et sols graveleux). Ces variations sont résumées dans les tableaux IV et V.

Tableau IV

\begin{tabular}{|c|c|c|}
\hline \multicolumn{2}{|c|}{ Pourcentage } & \multirow{2}{*}{ Passant } \\
\cline { 1 - 2 } Limons & Graves & \\
\hline $45 \%$ & $10 \%$ & $80 \Omega$ \\
$90 \%$ & $45 \%$ & $2 \mathrm{~mm}$ \\
$99 \%$ & $70 \%$ & $10 \mathrm{~mm}$ \\
$100 \%$ & $86 \%$ & $20 \mathrm{~mm}$ \\
$96 \%$ & $97 \%$ & $50 \mathrm{~mm}$ \\
\hline
\end{tabular}

\begin{tabular}{|c|c|c|c|}
\hline \multirow{7}{*}{ Tableau V } & \multicolumn{2}{|c|}{ Pourcentage } & \multirow{2}{*}{ Passant } \\
\hline & Limons sableux & Sables fins & \\
\hline & $24 \%$ & $5 \%$ & $80 \Omega$ \\
\hline & $50 \%$ & $38 \%$ & $1 \mathrm{~mm}$ \\
\hline & $58 \%$ & $48 \%$ & $2 \mathrm{~mm}$ \\
\hline & $90 \%$ & $80 \%$ & $10 \mathrm{~mm}$ \\
\hline & $100 \%$ & $98 \%$ & $15 \mathrm{~mm}$ \\
\hline
\end{tabular}

Les observations correspondant au 2ème cas concernent des différences granulométriques minimes quantitativement mais importantes pour le comportement des matériaux (fig.9).

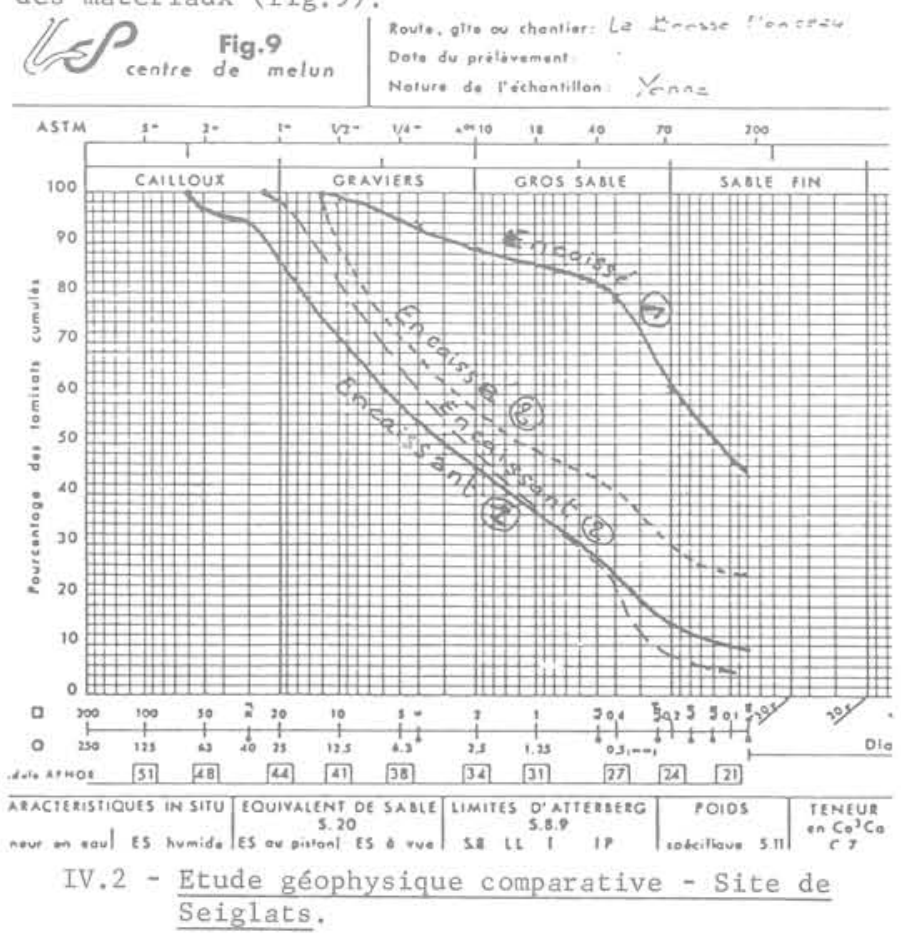

La comparaison des résultats obtenus par photo-interprétation et prospection géophysique s'appuie sur le site de Seiglats placé dans une boucle de l'YONNE à CANNES-ECLUSE (site $\mathrm{n}^{\circ} 2$, fig. 1).

La synthèse photogéologique a nécessité l'examen de 7 couvertures aériennes différentes et a permis :

- la cartographie lithologique (fig.10) et stratigraphique (fig.11) comprenant : des stries de glissement avec alternances graveleuses et limoneuses, des placages graveleux, des lits graveleux, des liserés argileux ;

- le dessin de la lithostratigraphie des dépots avec : les formations les plus anciennes au Sud et en position haute, le recoupement par des chenaux obliques à dominante graveleuse par délavage du matériau, des stries de glissement dans le lobe du méandre

corrélativement il a été dressé une carte géotechnique (fig.12). 
VALLEE DE IYONNE_CANNES - ECLUSE_Boucle des Seiglais" INTERPRETATION PHOTOGÉOLOGIOUE

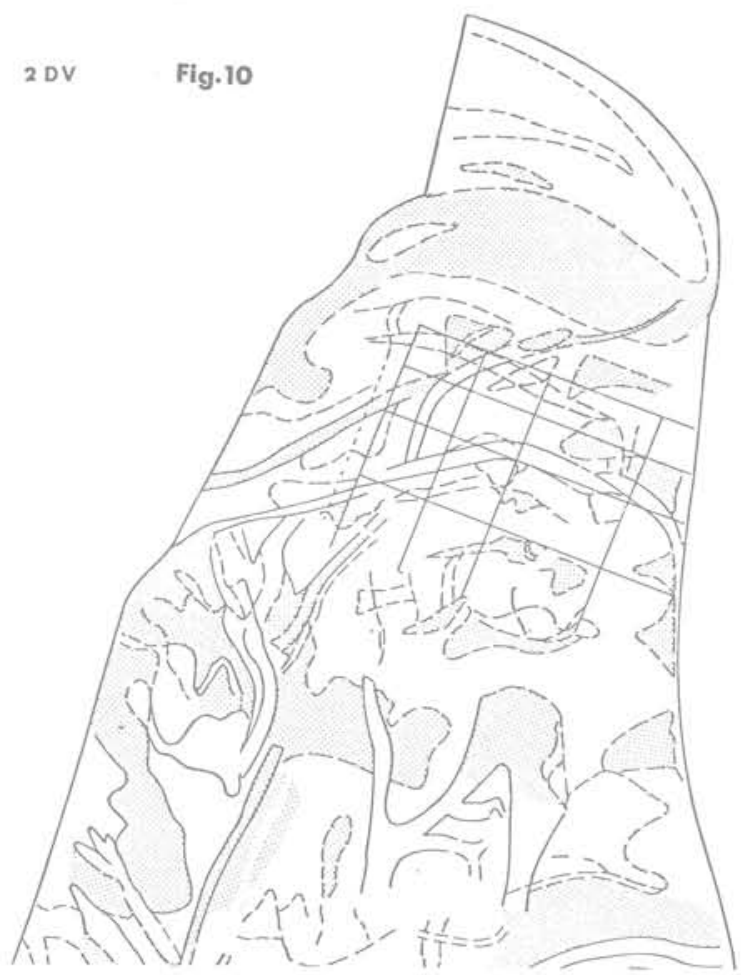

VALLEE DE L'YONNE_LANNES - CLLUSL_Boucle des Seiglats"

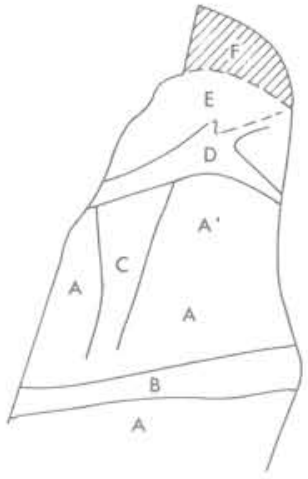

Fig:11

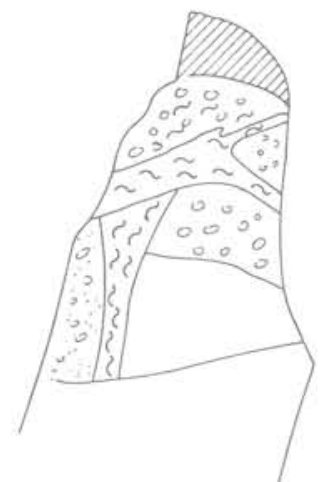

Fig.12
Stratigraphie des depots

Lo dynamique des depóls se reflete bien dans cette carte gui traduit lóge relotif des depóts flumous

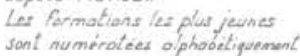
por ordre eroisiant.

$$
\begin{aligned}
& \text { Qualite geotechnique } \\
& \text { WVI stries de gliesement a dominante } \\
& \text { cr.: Stries de alissement Allernonce } \\
& 0: \text {, Materiaux arossiers } \\
& \text { IE } z \text { ane de chenaux } \\
& \text { - o Zone mixte }
\end{aligned}
$$

La prospection électrique repose sur plusieurs centaines de points mesurés par trainé avec une longueur de ligne $\frac{A B}{2}=10 \mathrm{~m}$ (fig.13). Elle indique : une zone

Sud de faible résistivité $(<60 \Omega / \mathrm{m})$ traversant la totalité du site, un pâle central de forte résistivité, deux alignements, I'un Nord-Sud de faible résisti- vité $(<80 \Omega)$ et l'autre oblique NE-SE à double résistivité faible vers le nord et très forte vers le Sud. Une zone à forte résistivité au Nord, bordée à proximité du fleuve par des terrains moins résistants, quelques zones isolées à forte résistivité relative $(>130 \Omega \mathrm{m}$ ).

VALLEE DE I'YONNE_CANNES - ECLUSE_Boucle des Seiglats CARTE D'ISORESISTIVITE AB/2 $=10 \mathrm{~m}$

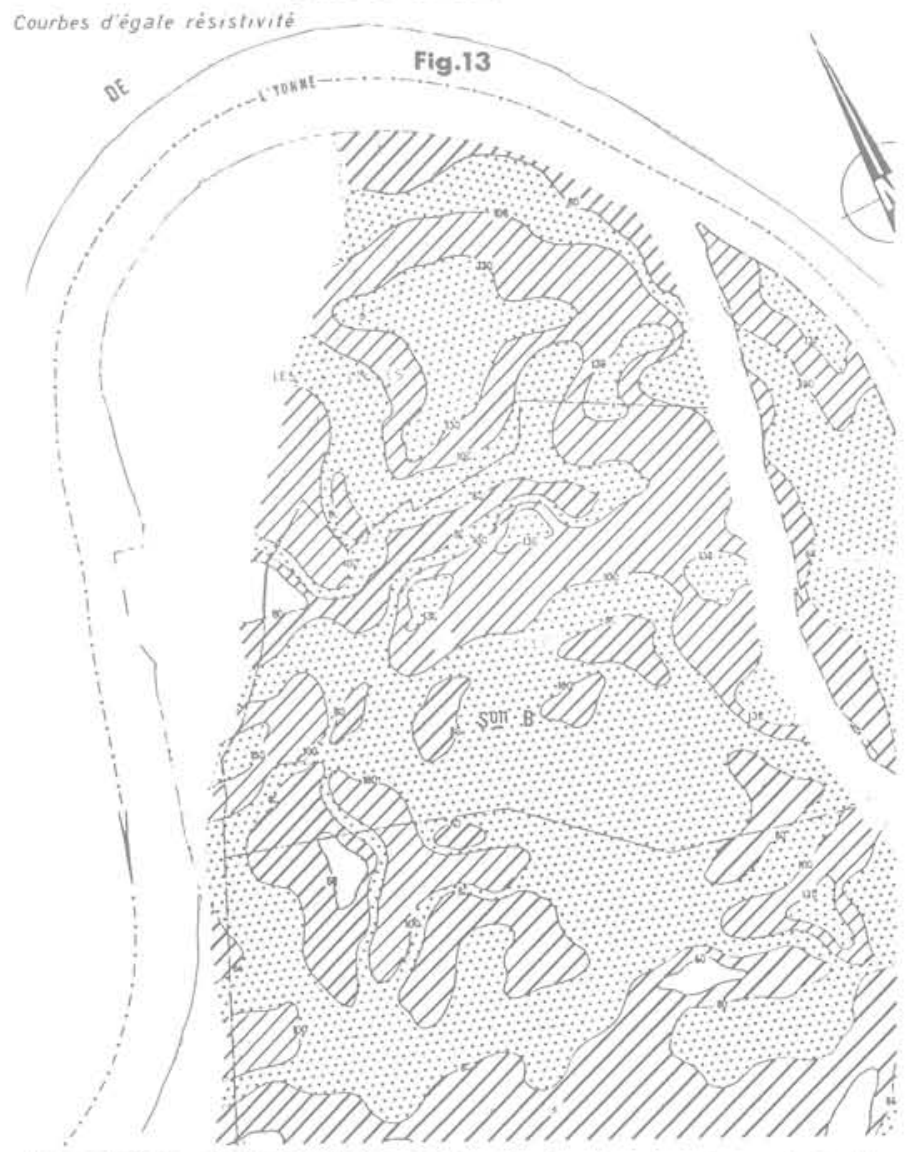

Le schéma géotechnique issu de la carte des résistivités apparentes s'avère très proche de la carte géotechnique élaborée par photo-interprétation (fig.14), Dans chacun de ces documents on retrouve les mêmes principaux éléments structurants. Toutefois, la photo-interprétation apparaît plus pessimiste dans la partie Nord du gisement avec des placages argileux plus étendus au niveau des stries de glissement. Elle semble, par contre, plus précise dans le dessin des chenaux d'écoulement tant pour leur géométrie que pour la nature de leur dépôt. Il s'agit là probablement d'un effet d'échelle en raison de la taille de la maille de prospection. Cependant, il est intéressant de noter que pour une longueur de ligne de $\mathrm{AB} / 2=6 \mathrm{~m}$, les résultats produits par géophysique paraissent moins représentatifs. Cela pourrait signifier que la photographie aérienne livre des informations sur les 2 ou 3 premiers mètres du sol. C'est d'ailleurs ce que confirme la comparaison faite avec la carte du toit du gisement graveleux au moyen de sondages mécaniques de vérification.

En définitive, les résultats provenant de la photointerprétation apportent une aide appréciable à la reconnaissance géotechnique des sites en autorisant I'allègement de la campagne de prospection géophysique dont le rôle essentiel serait celui du calage des indices révélés par la photo-interprétation. 
VALLEE DE I'YONNE_CANNES - ECLUSE_Boucle" des Sciglals"

B

CARTE DE REPRESENTATIVITE. INTERPRETATION PHOTOGRAPHIOUE ET ISORESISTIVITE

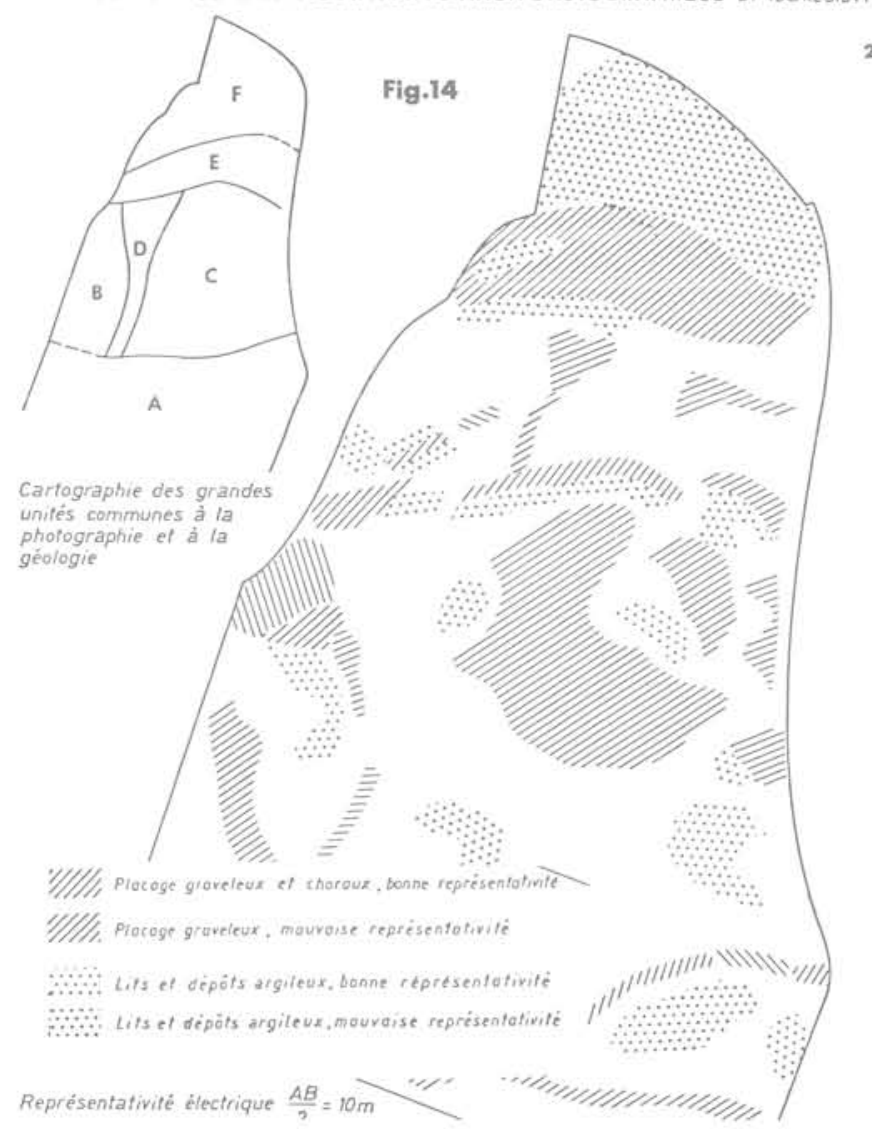

\section{$\mathrm{V}$ - CONCLUSIONS}

Il est clair que la photo-interprétation appliquée à la reconnaissance gétechnique et anthropique ne saurait être pertinente par 1'exploitation d'une seule mission fut-elle la plus récente. C'est au contraire $1^{\prime}$ analyse systématique des clichés pris à des époques différentes qui assure, par complémentarité des informations qu'ils fournissent, les meilleures chances $d^{\prime} u n$ recensement sinon exhaustif, du moins approfondi d'indices fugaces que seule peut révéler la lecture de 1'environnement paysager et cultural dans des conditions climatiques variées. Cela est particulièrement vrai à partir des missions les plus anciennes du fait des exigences du monde moderne qui. en modifiant le paysage gomment du même coup ses relations visuelles avec 1 'hétérogénéité des sols. En outre, la photo-interprétation multitemporelle, méthode peu coateuse, par la connaissance générale qu'elle donne des indices décelés, concoure au choix des périodes favorables des prises de vues obliques à basse altitude sur des secteurs restreints pour $1 \mathrm{e}-$ quel leur étude de détail s'impose. Périodes, dates et heures d'observation, altitude, angle de prises de vues, éclairement, vent et précipitations, conditionnent l'intérêt de ce type de prospection. Ce sont là des éléments qui, pour certains régissent des phénomènes physiques, biophysiques ou anthropiques et, pour d'autres, en accentuent 1'existence. Mais tous, contribuent de façon fondamentale au repérage aérien dans le paysage des anomalies qui en résultent. Autant de paramètres dont le choix peut être guidé par la consultation des archives photographiques de I'I.G.N. et le suivi régulier des données météorolo- giques et climatiques locales. Ainsi les prises de vues obliques à basse altitude conserveront toute leur efficacité par une souplesse de mise en oeuvre assurant le respect des conditions, au préalable déterminées, les plus propices à 1'examen et à la compréhension de détail du cadre physique dans lequel viendra s'inscrire le projet.

Associées de la sorte, photographies aériennes verticales et obliques apportent par la pertinence de leurs réponses la connaissance d'ensemble des sites nécessaire mais suffisante à $1^{\prime}$ organisation d'une campagne de terrain réduite alors pour l'essentiel à une prospection ponctuelle.

Si le développement de l'imagerie spatiale laisse entrevoir, aux spécialistes des sciences de la terre, aux photo-interprètes de diverses disciplines et aux aménageurs d'une façon générale, de très riches possibilités d'investigation globale des sites à petite échelle, la photographie aérienne classique verticale et oblique devrait néanmoins être appelée à jouer un rôle indispensable de guide pour la sélection et I'exploitation de ces nouvelles images.

\section{AGACHE R. (1966)}

$$
\text { BIBLIOGRAPHIE }
$$

Recherche des moments favorables à la mise en évidence des vestiges archéologiques arasés par l'agriculture dans le Nord de la FRANCE, Actes IIe Symp. int, photo-interprétation. PARIS

AUBERT J,, DORIDOT M., RICK P. (1979)

La photographie aérienne oblique. Une technique légère appliquée à la géologie de génie-civil et aux études d'environnement. Bull. liaison Ponts et Chaussées $n^{\circ} 3$ sept-oct 1979

AUBERT J., DORIDOT M., RICK P. (1980) Apport de la photographie aérienne appliquée à l'étude de gisements alluvionnaires, complémentarité avec la géophysique, utilisation de la télédétection - rapport interne L.R.E.P.

AUBERT J., GROS G. (1980)

La photo-interprétation multitemporelle et les prises de vues aériennes obliques à basse altitude - rapport L.R.E.P.-S.E.T.R.A.

CHEVALIER R. (1964)

L'avion à la découverte du passé, Fayard.

DASSIE J.(1978)

Manuel d'archéologie aérienne, Technip. PARIS.

GOGUEY R. (1966)

Recherche sur 1'influence des dates et des conditions de prises de vues de la détection aérienne des indices d'origine archéologique. Actes IIe Symp. int. photo-interprétation II.PARIS.

GROS G. (1980)

Quelques réponses aux besoins des ingénieurs routiers, revue de 1'association française de topographie $\mathrm{n}^{\circ} 4$ septembre.

JALMAIN D. (1970)

Archéologie aérienne en ILE-de-FRANCE, Technip.1.

STEINER D. (1966)

Investigation of seasonality as a factor affecting the photo-interpretation of rural land use. Actes IIe symp.int.photo-interprétation II, PARIS. 